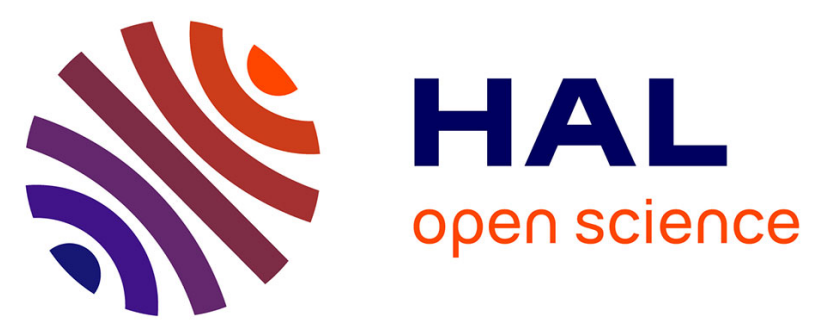

\title{
Exposition aux contraintes psychosociales en milieu de travail : résultats de l'enquête SUMER 2003 dans les Pays-de-la-Loire
}

Aurélie François, Catherine Ha, D. Waltisperger, Stéphane François, Serge Fanello, Yves Roquelaure

\section{To cite this version:}

Aurélie François, Catherine Ha, D. Waltisperger, Stéphane François, Serge Fanello, et al.. Exposition aux contraintes psychosociales en milieu de travail : résultats de l'enquête SUMER 2003 dans les Pays-de-la-Loire. Archives des Maladies Professionnelles et de L'Environnement, 2011, 72 (4), pp.333 - 340. 10.1016/j.admp.2011.07.003 . hal-03389576

\section{HAL Id: hal-03389576 \\ https://univ-angers.hal.science/hal-03389576}

Submitted on 21 Oct 2021

HAL is a multi-disciplinary open access archive for the deposit and dissemination of scientific research documents, whether they are published or not. The documents may come from teaching and research institutions in France or abroad, or from public or private research centers.
L'archive ouverte pluridisciplinaire HAL, est destinée au dépôt et à la diffusion de documents scientifiques de niveau recherche, publiés ou non, émanant des établissements d'enseignement et de recherche français ou étrangers, des laboratoires publics ou privés. 


\section{Exposition aux contraintes psychosociales en milieu de travail : résultats de l'enquête SUMER 2003 dans les Pays-de-la-Loire}

Exposure to psychosocial factors at work: Results of the
SUMER 2003 survey in the Pays-de-la-Loire region

A. François ${ }^{a, *}$, C. Ha ${ }^{b}$, D. Waltisperger ${ }^{c}$, S. François ${ }^{d}$, S. Fanello ${ }^{a}$, Y. Roquelaure ${ }^{d}$

a Département de santé publique, $\mathrm{CHU}, 4$, rue Larrey, 49033 Angers cedex, France

${ }^{b}$ Département santé travail, institut de veille sanitaire, 12, rue du Val-d'Osne, 94415 SaintMaurice cedex, France

${ }^{\mathrm{C}}$ Direction de l'animation de la recherche, des études et des statistiques (DARES), 39-43, quai André-Citroën, 75902 Paris cedex 15, France

'Laboratoire d'ergonomie et d'épidémiologie en santé au travail, CHU, 49033 Angers cedex, $\checkmark$ France
Disponible en ligne sur

\section{$\because$ ScienceDirect \\ www.sciencedirect.com}

\section{Résumé}

\section{Summary}

Aim of the study. The SUMER national survey assesses the exposure to major professional risks in France. The objective of this work is to describe the results of the exposure to psychosocial factors at work in the SUMER survey 2003 in the Pays-de-la-Loire region. Method. The survey was conducted from June 2002 to end 2003. The three factors elaborated by Karasek (psychological demands, decision latitude and social support) were assessed with the French version of the "Job Content Questionnaire" (JCQ) developed by Karasek.

Results. One thousand nine hundred and thirty-five workers were included (1110 men, 825 women), representative of the region's workforce after adjustment by calibration on margins. Twenty-six percent of the workers are exposed to situations of "job strain". These, combined or not with social isolation, are particularly common in industries with Taylorized work organization but also in business services and transport.

Discussion. The study shows that "job strain" and "iso strain" concern some occupational categories, particularly a large number of employees and blue-collar workers. However, psychic suffering at work have spread in recent years throughout the world of work and affect all socioprofessional categories. A preventive approach in the context of "work stress" implies an action on work organization.
Objectif. L'enquête SUMER permet une évaluation des expositions des salariés aux principaux risques professionnels en France. Sont présentés ici les résultats sur les contraintes psychosociales issus de l'enquête SUMER 2003 pour la région des Pays-de-la-Loire.

Méthode. L'enquête s'est déroulée de juin 2002 à fin 2003. Les trois dimensions psychosociales du modèle de Karasek (demande psychologique, latitude décisionnelle et soutien social) ont été évaluées à l'aide de la version française du «Job Content Questionnaire » (JQC).

Résultats. Pour les Pays-de-la-Loire, 1935 salariés ont été inclus (1110 hommes et 825 femmes), représentatifs des salariés de la région après redressement par calage sur marges. Vingt-six pourcent des salariés sont exposés à une forte demande psychologique en disposant d'une faible latitude décisionnelle pour y faire face. Les situations dites de «tension au travail », associées ou non à un manque de soutien social des collègues ou de la hiérarchie, sont particulièrement fréquentes dans les secteurs industriels fortement taylorisés (industrie des biens de consommation, agroalimentaire, biens intermédiaires), mais aussi dans les services aux entreprises et les transports.

Discussion. L'étude montre que les situations de «tension au travail » et de «tension au travail avec isolement social » concernent davantage certaines catégories socioprofessionnelles, en particulier

\footnotetext{
* Auteur correspondant.

e-mail : au.francois@hotmail.fr
} 
Conclusion. This survey, using a collective approach, contributes to take preventive measures in work organization.

(c) 2011 Elsevier Masson SAS. All rights reserved.

Keywords: Job strain, Work, Work organization, Prevention

\section{Introduction}

Le " stress au travail " constitue un sujet de préoccupations majeur en santé au travail. De nombreuses études tentent de mesurer le phénomène. L'approche statistique permet d'évaluer la prévalence de certaines contraintes pouvant retentir sur l'état de santé des travailleurs [1]. Au sein de l'Union européenne, $22 \%$ des salariés se déclarent "stressés " selon les résultats de la quatrième enquête sur les conditions de travail dans l'Union européenne en 2005 [2]. En France, le développement d'une politique de prévention des risques psychosociaux constitue un des objectifs principaux du deuxième plan santé au travail pour 2010-2014.

La recherche des causes du stress au travail implique d'étudier l'organisation du travail. Sur le plan épidémiologique, un modèle permettant d'explorer des éléments organisationnels, particulièrement utilisé est celui de "demande - latitude " développé par Robert Karasek et Töres Theorell [3,4]. Selon ce modèle, une situation de travail combinant une forte demande psychologique et une faible latitude décisionnelle augmente le risque de développer un problème de santé physique ou mentale [4]. Le score de demande psychologique porte sur la quantité de travail, la charge mentale et les contraintes de temps liées au travail. La latitude décisionnelle correspond à la possibilité de prendre des décisions dans le déroulement de son travail, mais aussi d'être créatif et d'utiliser et développer ses compétences. Plusieurs études ont confirmé l'hypothèse de Karasek en démontrant que le manque de contrôle sur une situation de travail qui présente de fortes exigences accroît le risque de détresse psychologique $[5-7]$ et de maladies physiques telles que les maladies cardiovasculaires $[3,4,8-10]$ et les troubles musculo-squelettiques (TMS) [11-13]. Ce modèle a ensuite été complété par une troisième dimension, le soutien social, qui renvoie aux interactions sociales au travail apportant une aide à l'individu, tant de la part des collègues que des supérieurs hiérarchiques. les ouvriers, les employés du commerce, les agents de la fonction publique et les employés administratifs. Cependant, les phénomènes de souffrance psychique au travail se sont diffusés ces dernières années à l'ensemble du monde du travail et touchent toutes les catégories socioprofessionnelles. Une démarche de prévention dans le cadre du « stress au travail » implique une action sur l'organisation du travail.

Conclusion. Cette étude, en évaluant l'exposition à certains facteurs de stress professionnel, montre l'intérêt d'une approche par l'organisation du travail dans une démarche de prévention.

(C) 2011 Elsevier Masson SAS. Tous droits réservés.

Mots clés : Tension au travail, Travail, Organisation du travail, Prévention

L'enquête SUMER 2002-2003 permet une évaluation des expositions des salariés aux principaux risques professionnels en France. Elle a été menée conjointement par la Direction des relations du travail (Inspection médicale du travail) et la Direction de l'animation de la recherche, des études et des statistiques (Dares). Cette enquête a été réalisée auprès d'un grand nombre de salariés surveillés par la médecine du travail, sauf ceux des fonctions publiques d'État et territoriale, d'une partie des transports, de France Telecom, des mines, de la pêche... Elle s'est déroulée sur le terrain de juin 2002 à fin 2003. En France, 1792 médecins du travail, soit plus de $20 \%$ des médecins du travail en exercice, ont tiré au sort 56314 salariés, dont 49984 ont répondu. Un auto-questionnaire comprenant le Job Content Questionnaire (JCO) de Karasek a été proposé à un salarié sur deux et 24486 personnes y ont répondu. La région Pays-de-la-Loire ainsi que l'île-de-France et la région Rhône-Alpes peuvent disposer de résultats régionaux du fait du nombre suffisant de questionnaires [14]. L'objectif de ce travail est de décrire les résultats du questionnaire de Karasek observés dans les Pays-de-la-Loire.

\section{Population et méthode}

\section{Recueil des données}

Pour les Pays-de-la-Loire, 178 médecins du travail, soit plus de $20 \%$ des médecins du travail en exercice, ont tiré au sort 4027 salariés, parmi lesquels 1935 ont répondu à l'auto-questionnaire.

Le JCO, dans sa version à 26 questions validée en français [8], a été utilisé pour évaluer la demande psychologique, la latitude décisionnelle et le soutien social. Les réponses aux 26 questions du questionnaire sont codées 1 ("pas du tout d'accord"), 2 (" pas d'accord"), 3 ("d'accord") ou 4 (" tout à fait d'accord"). Les scores de demande psychologique, de latitude décisionnelle et de soutien social ont été dichotomisés respectivement à 21, 
70 et 23 (correspondant aux médianes observées pour l'ensemble de la population de SUMER national) pour définir la forte demande psychologique, la faible latitude décisionnelle et le faible soutien social. Les situations dites de " tension au travail " correspondent à l'association d'une demande psychologique forte et d'une latitude décisionnelle faible. Lorsqu'il s'y ajoute un faible soutien social, il s'agit de la situation la plus péjorative, dite de "tension au travail avec isolement social".

\section{Analyse statistique}

La structure de l'échantillon des répondants n'est pas exactement la même que celle de la population salariée de la région, qui est ici notre population de référence. Pour redresser l'échantillon, une pondération régionale a été réalisée par calage sur marges à l'aide de la macro CALMAR de SAS développée par l'Insee, qui affecte aux réponses de chaque salarié un poids permettant de retrouver au niveau global les structures de la population de référence (par sexe, âge, catégorie socioprofessionnelle, secteur d'activité et taille d'établissement).

Les secteurs ont été regroupés selon la nomenclature économique de synthèse (NES 16) de l'Insee. Les professions ont été codées à l'aide de la nomenclature des professions et catégories socioprofessionnelles (PCS 1994) de l'Insee. Lorsque les effectifs par secteur économique et profession sont inférieurs à dix, les résultats ne figurent pas dans les tableaux.

\section{Résultats}

L'échantillon est constitué de 1935 salariés, soit 1110 hommes et 825 femmes, représentant 1,7 pour mille salariés des Paysde-la-Loire (1,8\%o des salariés hommes et 1,6\%o des salariées femmes). L'âge moyen est de 38,2 ans (ET $=10,6)$. On observe une légère sous-représentation des femmes par rapport aux données régionales : $43 \%$ versus $46 \%$ (tableau I).

Au niveau de l'emploi, on observe une surreprésentation de l'industrie manufacturière et agroalimentaire. Concernant les catégories socioprofessionnelles, les ouvriers sont surreprésentés et les cadres sous-représentés. Les salariés sont principalement en contrat à durée indéterminée $(84,4 \%)$, plus rarement intérimaires ( $4,3 \%)$, fonctionnaires (3,9\%), en contrat à durée déterminée (3,8\%) ou apprentis ou stagiaires (3,6 \%) (tableau II).

\section{Exposition aux facteurs psychosociaux}

\section{Scores de Karasek}

Les scores extrêmes ainsi que les scores moyens (pondérés) des trois dimensions du JCQ sont :

- demande psychologique : 9 à 36 , score moyen $=21,3$ $(\mathrm{ET}=4,2)$;

- latitude décisionnelle : 24 à 96 , score moyen $=70,5$ (ET = 11,7) ;

- soutien social : 8 à 32, score moyen $=23,9(E T=3,5)$.

\section{Demande psychologique élevée}

L'exposition à une forte demande psychologique concerne $55 \%$ de l'ensemble des salariés. Les femmes sont davantage concernées que les hommes (tableau III).

Les secteurs où la demande est le plus fréquemment élevée sont les industries de biens de consommation, les activités financières, les services aux entreprises, l'éducation, la santé et l'action sociale, l'agroalimentaire et les industries des biens intermédiaires (tableau IV). Les professions les plus exposées sont les ingénieurs et cadres techniques d'entreprise, ainsi que les cadres administratifs et commerciaux d'entreprise (tableau V).

\section{Latitude décisionnelle faible}

Quarante-neuf pourcent des salariés ont une faible latitude décisionnelle pour faire face aux contraintes des situations de travail. L'exposition à une faible latitude décisionnelle est nettement plus fréquente chez les intérimaires, puisque trois quarts d'entre eux sont concernés. Les femmes sont davantage concernées que les hommes (tableau III).

Les résultats montrent que les secteurs particulièrement concernés sont les industries de biens de consommation, l'agroalimentaire, les transports, les services aux particuliers, les services aux entreprises ainsi que les industries des biens intermédiaires (tableau IV). Les ouvriers non qualifiés de type industriel, les manutentionnaires, les chauffeurs, les ouvriers non qualifiés de type artisanal, les personnels de services directs aux particuliers, les ouvriers qualifiés de type industriel et les employés sont les plus exposés (tableau V).

Tableau I

Caractéristiques de l'échantillon de salariés ayant répondu à l'auto-questionnaire, SUMER 2003, Pays-de-la-Loire.

\begin{tabular}{|c|c|c|c|c|c|}
\hline & $\begin{array}{l}\text { Échantillon } \\
\text { de salariés }\end{array}$ & Effectif (\%) & $\begin{array}{l}\text { Population } \\
\text { salariée }^{a}\end{array}$ & Effectif (\%) & $\begin{array}{l}\% \text { de salariés inclus pour } \\
1000 \text { salariés }^{\mathrm{a}}\end{array}$ \\
\hline Hommes & 1110 & 57 & 600713 & 54 & 1,8 \\
\hline Femmes & 825 & 43 & 519098 & 46 & 1,6 \\
\hline Total & 1935 & 100 & 1119811 & 100 & 1,7 \\
\hline
\end{tabular}

${ }^{a}$ Population salariée, âgée de 15 à 60 ans ou plus, des Pays-de-la-Loire (recensement Insee du 9/3/1999). 
Tableau II

Caractéristiques sociodémographiques et professionnelles de l'échantillon, SUMER 2003, Pays-de-la-Loire.

\begin{tabular}{|c|c|c|c|c|c|c|}
\hline & \multicolumn{2}{|c|}{$\begin{array}{l}\text { Échantillon total } \\
n=1935\end{array}$} & \multicolumn{2}{|c|}{ Hommes $n=1110$} & \multicolumn{2}{|c|}{ Femmes $n=825$} \\
\hline & $n$ & $\%$ & $n$ & $\%$ & $n$ & $\%$ \\
\hline \multicolumn{7}{|l|}{ Âge } \\
\hline$<25$ ans & 225 & 13,0 & 130 & 13,3 & 95 & 12,6 \\
\hline $25-29$ ans & 265 & 13,4 & 158 & 13,7 & 107 & 12,9 \\
\hline $30-39$ ans & 551 & 28,8 & 318 & 28,8 & 233 & 28,9 \\
\hline $40-49$ ans & 546 & 26,4 & 301 & 25,4 & 245 & 27,6 \\
\hline 50 ans et plus & 348 & 18,4 & 203 & 18,8 & 145 & 17,9 \\
\hline \multicolumn{7}{|l|}{ Type de contrat de travail } \\
\hline Fonctionnaire & 84 & 3,9 & 19 & 1,3 & 65 & 7,4 \\
\hline CDI & 1651 & 84,4 & 982 & 87,4 & 669 & 80,4 \\
\hline CDD & 66 & 3,8 & 21 & 2,3 & 45 & 5,8 \\
\hline Intérimaire & 75 & 4,3 & 54 & 5,2 & 21 & 3,1 \\
\hline Apprenti ou stagiaire & 59 & 3,6 & 34 & 3,9 & 25 & 3,3 \\
\hline \multicolumn{7}{|l|}{ Secteur d'activité (NAF) } \\
\hline Agriculture & 10 & & 7 & & 3 & \\
\hline Industrie & 703 & 29,4 & 485 & 35,6 & 218 & 20,9 \\
\hline Construction & 103 & 8,3 & 98 & 13,9 & 5 & 0,8 \\
\hline Tertiaire & 1119 & 62,3 & 520 & 50,5 & 599 & 78,3 \\
\hline \multicolumn{7}{|c|}{ Catégorie socioprofessionnelle (PCS) } \\
\hline Cadres & 181 & 8,8 & 129 & 10,7 & 52 & 6,1 \\
\hline Professions intermédiaires & 491 & 21,8 & 291 & 21,7 & 200 & 22,0 \\
\hline Employés & 472 & 25,5 & 89 & 8,3 & 383 & 48,9 \\
\hline Ouvriers & 791 & 43,9 & 601 & 59,3 & 190 & 23,0 \\
\hline
\end{tabular}

$n$ : effectifs dans l'échantillon ;\% : pourcentage de la catégorie en données pondérées.

\section{Soutien social faible}

Le soutien social, des collègues ou de l'encadrement, est faible pour $41 \%$ des salariés, plus souvent pour les femmes que pour les hommes, sans que la différence soit statistiquement significative (tableau III).
Les secteurs d'activité les plus touchés sont l'énergie et l'industrie de biens de consommation (tableau IV). Un faible soutien social est observé plus fréquemment chez les ouvriers non qualifiés de type industriel, les manutentionnaires, les ouvriers qualifiés de type industriel, les employés civils et agents de service de la fonction publique et les professions

\section{Tableau III}

Proportion (\%) de salariés exposés aux principales contraintes psychosociales au travail en fonction du genre et du contrat de travail, SUMER 2003, Pays-de-la-Loire.

\begin{tabular}{|c|c|c|c|c|c|}
\hline & $\begin{array}{l}\text { Demande } \\
\text { psychologique } \\
\text { élevée }\end{array}$ & $\begin{array}{l}\text { Latitude } \\
\text { décisionnelle } \\
\text { faible }\end{array}$ & $\begin{array}{l}\text { Soutien } \\
\text { social faible }\end{array}$ & $\begin{array}{l}\text { Tension au } \\
\text { travail }\end{array}$ & $\begin{array}{l}\text { Tension avec isolement } \\
\text { social au travail }\end{array}$ \\
\hline \multicolumn{6}{|l|}{ Genre } \\
\hline Hommes (1110) & 52 & 43 & 39 & 21 & 12 \\
\hline Femmes (825) & 58 & 58 & 44 & 34 & 21 \\
\hline \multicolumn{6}{|l|}{ Contrat de travail } \\
\hline Fonctionnaire (84) & 61 & 50 & 42 & 29 & 17 \\
\hline CDI (1651) & 56 & 47 & 43 & 26 & 16 \\
\hline CDD (66) & 51 & 57 & 36 & 28 & 12 \\
\hline Intérimaire (75) & 42 & 74 & 24 & 36 & 13 \\
\hline Apprenti (59) & 41 & 61 & 34 & 22 & 11 \\
\hline $\begin{array}{l}\text { Ensemble (1935) } \\
\quad \text { IC } 95 \%\end{array}$ & $\begin{array}{l}55 \\
(52,8-57,2)\end{array}$ & $\begin{array}{l}49 \\
(46,8-51,2)\end{array}$ & $\begin{array}{l}41 \\
(38,8-43,2)\end{array}$ & $\begin{array}{l}26 \\
(24,0-28,0)\end{array}$ & $\begin{array}{l}16 \\
(14,4-17,6)\end{array}$ \\
\hline
\end{tabular}

Tension au travail : demande psychologique élevée et latitude décisionnelle faible; tension avec isolement social au travail : demande psychologique élevée, latitude décisionnelle faible et soutien social faible. 
Tableau IV

Proportion (\%) de salariés exposés aux principales contraintes psychosociales au travail en fonction du secteur d'activité, SUMER 2003, Pays-de-la-Loire.

\begin{tabular}{llllll} 
Secteur économique (NES 16) & $\begin{array}{l}\text { Demande } \\
\text { psychologique } \\
\text { élevée }\end{array}$ & $\begin{array}{l}\text { Latitude } \\
\text { décisionnelle } \\
\text { faible }\end{array}$ & $\begin{array}{l}\text { Soutien } \\
\text { social } \\
\text { faible }\end{array}$ & $\begin{array}{l}\text { Tension au } \\
\text { travail }\end{array}$ & $\begin{array}{l}\text { Tension avec } \\
\text { isolement social } \\
\text { au travail }\end{array}$ \\
\hline Agriculture (10) & - & - & - & - & - \\
Agroalimentaire (139) & 55 & 59 & 41 & 32 & 19 \\
Industries de biens de consommation (129) & 66 & 67 & 53 & 45 & 32 \\
Industrie automobile (19) & 46 & 49 & 46 & 17 & 14 \\
Industries de biens d'équipement (213) & 54 & 44 & 36 & 23 & 10 \\
Industries des biens intermédiaires (177) & 55 & 53 & 44 & 29 & 16 \\
Energie (26) & 29 & 46 & 61 & 0 & 0 \\
Construction (103) & 40 & 31 & 31 & 11 & 5 \\
Commerce (340) & 53 & 45 & 41 & 23 & 16 \\
Transports (78) & 53 & 58 & 36 & 31 & 17 \\
Activités financières (78) & 66 & 38 & 31 & 27 & 12 \\
Activités immobilières (22) & 49 & 26 & 38 & 35 & 0 \\
Services aux entreprises (241) & 62 & 57 & 45 & 28 & 17 \\
Services aux particuliers (77) & 44 & 58 & 47 & 24 & 15 \\
Éducation, santé, action sociale (229) & 60 & 44 & 41 & 15 & 10 \\
Administrations (54) & 46 & 49 & 49 & 26 & 16 \\
Ensemble (1935) & 55 & $(46,8-51,2)$ & $(38,8-43,2)$ & $(24,0-28,0)$ & $(14,4-17,6)$
\end{tabular}

Les résultats ne figurent pas pour les secteurs dont les effectifs observés sont inférieurs à dix.

intermédiaires administratives et commerciales d'entreprise (tableau V).

\section{Situation de " tension au travail " ou " job strain "}

Vingt-six pourcent des salariés sont exposés à une forte demande psychologique en disposant d'une faible latitude décisionnelle pour y faire face. Cette situation touche davantage les femmes que les hommes et est plus fréquente chez les intérimaires, $36 \%$ d'entre eux étant concernés (tableau III). Elle est particulièrement fréquente dans l'industrie de biens de consommation, les services aux entreprises, l'agroalimentaire, les transports, et l'industrie des biens intermédiaires (tableau IV). Les professions les plus exposées sont les ouvriers non qualifiés de type industriel, les ouvriers de la manutention, les employés de commerce, les employés civils et agents de service de la fonction publique, les employés administratifs d'entreprise, les ouvriers qualifiés de type industriel et les personnels de services directs aux particuliers (tableau V) (fig. 1).

\section{Situation de " tension au travail avec isolement social " ou " iso strain "}

Seize pourcent des salariés sont exposés au cumul des trois contraintes psychosociales. Cette situation, considérée comme la plus défavorable, concerne plus de femmes que d'hommes (tableau III).

Les secteurs les plus touchés sont l'industrie de biens de consommation, les services aux particuliers, l'agroalimentaire, les transports et les services aux entreprises (tableau IV). Les professions où ces situations sont retrouvées le plus fréquemment sont les ouvriers non qualifiés de type industriel, les manutentionnaires, les employés civils et agents de service de la fonction publique, les employés de commerce, les personnels de services directs aux particuliers ainsi que les ouvriers qualifiés de type industriel (tableau V) (fig. 1).

\section{Discussion}

L'enquête SUMER permet une approche épidémiologique de l'exposition des salariés aux principaux risques professionnels et, en particulier, à certains facteurs de risque de stress au travail. Le nombre suffisant de questionnaires dans les Paysde-la-Loire permet d'exploiter les résultats au niveau régional. La structure de l'échantillon n'étant pas exactement la même que celle de la population de référence, un redressement a été réalisé à l'aide d'un calage sur marges, en utilisant comme variables de calage le sexe, l'âge, la catégorie socioprofessionnelle, le secteur d'activité et la taille d'établissement. Les résultats présentés ici ne tiennent compte que d'un nombre limité de variables pouvant retentir sur l'état de santé mentale et physique des salariés. En effet, le questionnaire de Karasek n'évalue que certaines dimensions du travail. Bien d'autres facteurs peuvent également jouer un rôle, comme par exemple les contraintes liées au temps de travail, aux rythmes de travail ou le manque de reconnaissance du travail réel. 


\begin{tabular}{|c|c|c|c|c|c|}
\hline Professions (nomenclature PCS) & $\begin{array}{l}\text { Demande } \\
\text { psychologique } \\
\text { élevée }\end{array}$ & $\begin{array}{l}\text { Latitude } \\
\text { décisionnelle } \\
\text { faible }\end{array}$ & $\begin{array}{l}\text { Soutien } \\
\text { social } \\
\text { faible }\end{array}$ & $\begin{array}{l}\text { Tension } \\
\text { au } \\
\text { travail }\end{array}$ & $\begin{array}{l}\text { Tension avec } \\
\text { isolement social } \\
\text { au travail }\end{array}$ \\
\hline $\begin{array}{l}\text { Cadres fonction publique (hôpitaux publics, } \\
\text { La Poste) (3) }\end{array}$ & - & - & - & - & - \\
\hline Professeurs, professions scientifiques (4) & - & - & - & - & - \\
\hline $\begin{array}{l}\text { Professions de l'information, des arts, } \\
\text { spectacles (6) }\end{array}$ & - & - & - & - & - \\
\hline $\begin{array}{l}\text { Cadres administratifs et commerciaux } \\
\text { d'entreprise (95) }\end{array}$ & 72 & 10 & 37 & 7 & 2 \\
\hline $\begin{array}{l}\text { Ingénieurs et cadres techniques } \\
\text { d'entreprise (73) }\end{array}$ & 76 & 12 & 40 & 6 & 2 \\
\hline Instituteurs et assimilés (14) & 67 & 21 & 42 & 3 & 3 \\
\hline $\begin{array}{l}\text { Professions intermédiaires de la santé et } \\
\text { du travail social (103) }\end{array}$ & 68 & 34 & 38 & 18 & 10 \\
\hline $\begin{array}{l}\text { Professions intermédiaires administratives } \\
\text { de la FP (6) }\end{array}$ & - & - & - & - & - \\
\hline $\begin{array}{l}\text { Professions intermédiaires administratives } \\
\text { et commerciales d'entreprise (191) }\end{array}$ & 64 & 36 & 44 & 23 & 14 \\
\hline Techniciens (sauf tertiaire) (111) & 68 & 25 & 36 & 17 & 8 \\
\hline Contremaîtres et agents de maîtrise (66) & 63 & 23 & 42 & 17 & 17 \\
\hline $\begin{array}{l}\text { Employés civils et agents de service de la fonction } \\
\text { publique (84) }\end{array}$ & 47 & 54 & 45 & 32 & 24 \\
\hline Policiers et militaires (7) & - & - & - & - & - \\
\hline Employés administratifs d'entreprise (202) & 58 & 59 & 43 & 32 & 17 \\
\hline Employés de commerce (127) & 55 & 59 & 37 & 35 & 21 \\
\hline Personnels des services directs aux particuliers (52) & 40 & 60 & 39 & 31 & 21 \\
\hline Ouvriers qualifiés de type industriel (246) & 47 & 60 & 46 & 31 & 18 \\
\hline Ouvriers qualifiés de type artisanal (124) & 38 & 37 & 31 & 15 & 11 \\
\hline Chauffeurs (59) & 42 & 63 & 32 & 30 & 13 \\
\hline $\begin{array}{l}\text { Ouvriers qualifiés de la manutention, } \\
\text { du magasinage (78) }\end{array}$ & 62 & 65 & 47 & 38 & 24 \\
\hline Ouvriers non qualifiés de type industriel (198) & 52 & 80 & 47 & 44 & 27 \\
\hline Ouvriers non qualifiés de type artisanal (83) & 37 & 62 & 41 & 22 & 11 \\
\hline Ouvriers agricoles (3) & - & - & - & - & - \\
\hline $\begin{array}{l}\text { Ensemble (1935) } \\
\text { IC } 95 \%\end{array}$ & $\begin{array}{l}55 \\
(52.8-57.2)\end{array}$ & $\begin{array}{l}49 \\
(46.8-51.2)\end{array}$ & $\begin{array}{l}41 \\
(38.8-43.2)\end{array}$ & $\begin{array}{l}26 \\
(24.0-28.0)\end{array}$ & $\begin{array}{l}16 \\
(14.4-17.6)\end{array}$ \\
\hline
\end{tabular}

Les résultats ne figurent pas pour les professions dont les effectifs observés sont inférieurs à dix.

Les scores moyens de Karasek chez les salariés des Pays-de-laLoire sont proches de ceux obtenus au niveau national dans l'enquête SUMER $[15,16]$. Ils sont voisins de ceux calculés chez les salariés inclus dans la cohorte GAZEL [17] et de ceux retrouvés par le réseau de surveillance épidémiologique des TMS auprès de 1495 salariés dans les Pays-de-la-Loire inclus en 2002 [18].

Le manque de latitude décisionnelle concerne la moitié des salariés, et tout particulièrement, les ouvriers et employés. La latitude décisionnelle permet à l'opérateur un aménagement de son mode opératoire et joue un rôle majeur dans la possibilité de faire face aux contraintes de la tâche, pour pouvoir réaliser l'activité. Au niveau collectif, les marges de manœuvre sont nécessaires pour permettre une régulation des modes opératoires et de la répartition des tâches.
Les situations de "tension au travail " ou " job strain " touchent un salarié sur quatre. Associées ou non à un isolement social, elles sont particulièrement fréquentes dans les secteurs industriels fortement taylorisés, comme l'industrie des biens de consommation, l'agroalimentaire et l'industrie des biens intermédiaires, mais aussi dans les services aux entreprises et les transports. Elles concernent un grand nombre d'ouvriers, d'employés du commerce, d'agents de la fonction publique et d'employés administratifs. Ces situations exposent à un risque accru de pathologies psychiques et physiques. Elles sont aggravées par l'insuffisance ou l'absence de soutien social au travail [4].

Les notions de " job strain " et d' " iso strain ", définies par le modèle de Karasek, sont à différencier du terme de stress. En effet, les salariés dans ces situations ne sont pas les seuls à 


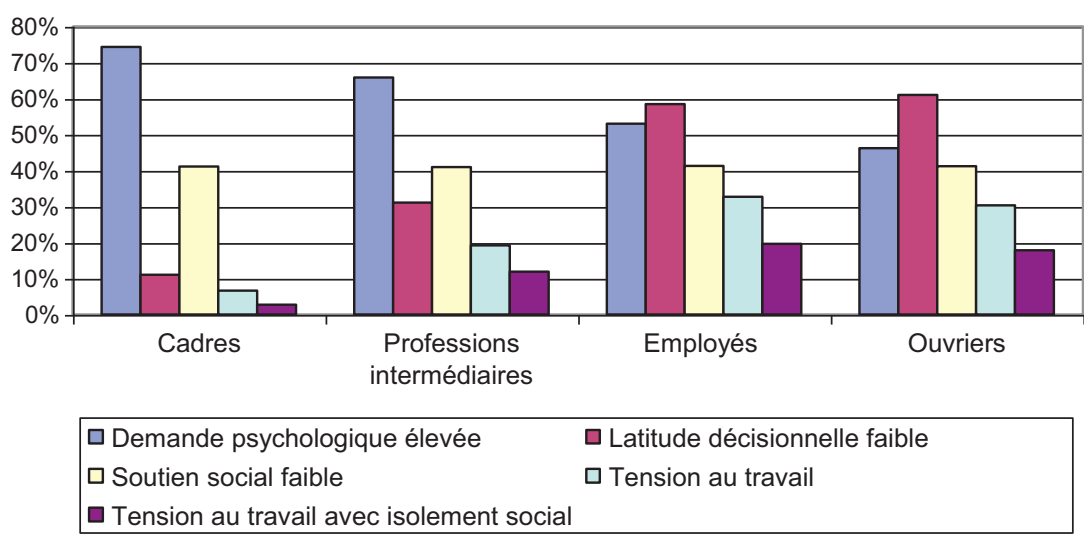

Figure 1. Proportion de salariés exposés aux principales contraintes psychosociales au travail en fonction de la catégorie socioprofessionnelle, SUMER 2003, Pays-de-la-Loire.

trouver leur travail stressant [14]. Par ailleurs, le terme de stress est extrêmement flou et est utilisé pour exprimer de façon globale les difficultés rencontrées, la souffrance au travail et ses conséquences sur l'état de santé [19]. L'utilisation de ce terme ne doit donc pas empêcher de " penser » la situation de travail pour pouvoir l'analyser. Par ailleurs, les situations de " job strain " et d'" iso strain " concernent davantage certaines catégories socioprofessionnelles. Cependant, les phénomènes de souffrance psychique au travail se sont diffusés ces dernières années à l'ensemble du monde du travail et touchent toutes les catégories socioprofessionnelles [20].

Une démarche de prévention dans le cadre du " stress au travail " implique une action sur l'organisation du travail. En effet, si l'organisation du travail est trop rigide concernant la répartition des tâches et les modes opératoires, elle ne laisse pas de marges de manœuvre suffisantes, individuelles et collectives [21]. Les marges de manœuvre offrent la possibilité aux opérateurs de pouvoir faire face aux contraintes et aux aléas, non prévus par l'organisation du travail, liés par exemple aux diverses exigences plus ou moins contradictoires du travail prescrit ou à la variabilité des situations de travail. En effet, il existe un écart irréductible entre travail prescrit et travail réel. Les opérateurs construisent ainsi un compromis pour l'élaboration de l'activité [22]. Ils mettent en jeu leur créativité et leur ingéniosité afin de combler les lacunes de l'organisation prescrite. Ces " ficelles de métier " ne sont rendues visibles collectivement que dans un contexte de confiance. En effet, les relations de confiance entre les sujets vont permettre la construction de liens de coopération. La notion de coopération renvoie à la volonté des personnes de travailler ensemble, de former un collectif de travail, ce qui permet l'établissement de "règles de métier " [22,23]. Ainsi, une démarche de prévention implique de fournir des marges de manœuvre, individuelles et collectives, suffisantes et de favoriser la mise en place des conditions nécessaires à l'établissement de relations de confiance entre les salariés. Au cours de ces deux dernières décennies, l'intensification du travail, avec l'accélération des cadences, la multiplication des contraintes sur les rythmes de travail, la diffusion de la production en flux tendu, l'augmentation conjointe des contraintes industrielles et marchandes [24], ont conduit à l'éclatement des collectifs. Les formes modernes de management participent également à ce phénomène en individualisant les salariés [1]. Les collectifs de travail peuvent aussi être mis à mal en cas de rotations ou de turn-over des opérateurs, ce qui empêche la mise en place des conditions nécessaires à la coopération. Les stratégies de régulation collective ne sont plus possibles. En conséquence, la démarche de prévention du " stress au travail " passe par la reconstruction d'une organisation du travail qui permette de restaurer des processus d'entraide et de favoriser le travail collectif par la coopération, en laissant par exemple aux salariés les moyens en termes de temps et d'espace pour échanger sur le travail. Concernant les nouvelles méthodes d'évaluation du travail, l'évaluation individualisée des performances entraîne une mise en concurrence des salariés avec une destruction de la confiance et des collectifs de travail $[22,25,26]$. De plus, les gestionnaires chargés de contrôler la qualité et d'évaluer les performances, ne connaissent pas le travail et ne peuvent contrôler que des données chiffrées ou des fiches techniques, bien loin du réel du travail et de ses difficultés éventuelles [25]. Ainsi, la question de l'évaluation du travail est également une piste dans la démarche de prévention du "stress professionnel " avec la prise en compte de critères relatifs à la réalité du travail et la participation des salariés eux-mêmes. La question de la reconnaissance comme rétribution, matérielle mais surtout symbolique, de la contribution du sujet à l'organisation du travail est essentielle $[23,25]$. Le fait de favoriser la reconnaissance du travail réel constitue un élément important dans la démarche de prévention. Par ailleurs, certaines formes de domination par le maniement managérial de la menace à la précarisation sont à l'origine de fortes tensions au travail. Le terme de précarisation souligne ici les conséquences de la précarité sur ceux qui travaillent : " c'est leur emploi qui est précarisé par le recours possible aux emplois précaires 
pour les remplacer, et aux licenciements pour le moindre écart " $[22,27]$. Ainsi, la menace du licenciement, l'incertitude sur le travail et sur l'emploi constituent autant de facteurs de fragilisation au travail.

\section{Conclusion}

Cette étude permet d'évaluer l'exposition à certains facteurs de stress professionnel, ainsi que d'identifier les secteurs d'activité et les catégories socioprofessionnelles particulièrement touchés. Une démarche de prévention implique d'aborder les questions du " stress " par le biais de l'organisation du travail. Le travail, en tant qu'activité humaine, c'est-à-dire avec tout l'engagement des individus que cela implique, est à replacer en position centrale des préoccupations. Cela demande d'intégrer dans une démarche de prévention ceux qui connaissent le travail réel, qui l'expérimentent physiquement et psychiquement : les travailleurs eux-mêmes.

\section{Déclaration d'intérêts}

Les auteurs déclarent ne pas avoir de conflits d'intérêts en relation avec cet article.

\section{Références}

[1] Gollac M, Volkoff S. Les conditions de travail. Paris: La découverte; 2000.

[2] Parent-Thirion A, Fernández Macías E, Hurley J, et al. Quatrième enquête européenne sur les conditions de travail 2005. Fondation européenne pour l'amélioration des conditions de vie et de travail. Luxembourg: Office des publications officielles des Communautés européennes; 2007.

[3] Karasek R. Job demands, job decision latitude, and mental strain: implications for job redesign. Adm Sci O 1979;24:285-308.

[4] Karasek R, Theorell T. Healthy work: stress, productivity and the reconstruction of the working life. New York: Basic Books; 1990

[5] Bourbonnais R, Brisson C, Vezina $M$, et al. Job strain and psychological distress in white-collar workers. Scand J Work Environ Health 1996;22:139-45.

[6] Chamoux A, Paris C, Desheulles J, et al. Stress et travail : rôle de la pénibilité psychique du travail. Résultats de l'enquête nationale multicentrique ASMT 96. Arch Mal Prof Environ 1999;60(6):570-1.

[7] Davezies P. Stress, pouvoir d'agir et santé mentale. Arch Mal Prof Environ 2008;69:195-203.

[8] Ishizaki M, Nakagawa H, Morikawa Y. Influence of job strain on changes in body mass index and waist circumference-6-year longitudinal study. Scand J Work Environ Health 2008;34:288-96.

[9] Landsbergis PA, Schnall PL, Belkić KL, et al. Work conditions and masked (hidden) hypertension-insights into the global epidemic of hypertension. Scand J Work Environ Health 2008; 6:41-51.

[10] Michikawa T, Nishiwaki Y, Nomiyama T, et al. Job strain and arteriosclerosis in three different types of arteries among male Japanese factory workers. Scand J Work Environ Health 2008;34:48-54.

[11] Hérisson C, Fouquet B, Codine P. Membre supérieur et pathologie professionnelle. Paris: Masson; 2001.

[12] Lasfargues $G$, Roquelaure $Y$, Fouquet $B$, et al. Pathologies ostéoarticulaires par hypersollicitation d'origine professionnelle. Paris: Masson; 2003

[13] Roquelaure $\mathrm{Y}, \mathrm{Ha} C$, Sauteron $M$. Réseau expérimental de surveillance des troubles musculo-squelettiques dans les Pays-de-la-Loire : surveillance en entreprise en 2002. Rapport de recherche, Institut de veille sanitaire, Saint-Maurice, 2005 (http://invs.sante.fr/publications).

[14] Guignon N, Niedhammer I, Sandret N. Les facteurs psychosociaux au travail. Une évaluation par le questionnaire de Karasek dans l'enquête SUMER 2003. DARES, Premières synthèses, premières informations, mai 2008, $\mathrm{n}^{\circ}$ 22.1.

[15] Niedhammer I, Chastang J-F, Gendrey L, et al. Propriétés psychométriques de la version française des échelles de la demande psychologique, de la latitude décisionnelle et du soutien social du " Job Content Ouestionnaire " de Karasek : résultats de l'enquête nationale SUMER. Sante Publique 2006;18:413-27.

[16] Niedhammer I, Chastang J-F, Levy D, et al. Exposition aux facteurs psychosociaux au travail du modèle de Karasek en France : étude méthodologique à l'aide de l'enquête nationale SUMER. Travailler 2007;17:47-70.

[17] Niedhammer I. Psychometric properties of the French version of the Karasek Job Content Questionnaire : a study of the scales of decision latitude, psychological demands, social support, and physical demands in the GAZEL cohort. Int Arch Occup Environ Health 2002;75:129-44.

[18] Roquelaure Y, Ha C, Gohier B, et al. Exposition au stress psychosocial chez les salariés des Pays-de-la-Loire en 2002. Encephale 2007;33:160-8.

[19] Davezies P. Le stress au travail : entre savoirs scientifiques et débat social. Perform Strateg Fact Hum 2001;1:4-7.

[20] Davezies P. Souffrance au travail : le risque organisationnel. Conférence introductive aux journées médicales du CISME sur le risque organisationnel. Publiée dans les actes ; février 2004.

[21] Guiho-Bailly MP, Guillet D. Psychopathologie et psychodynamique du travail. EMC - Toxicol-Pathol 2005;2(3):98-110.

[22] Dejours C. Travail, usure mentale. Paris: Bayard; 2008.

[23] Dejours C. Coopération et construction de l'identité en situation de travail. Congrès de la société d'ergonomie de langue française, septembre 1992, Lille.

[24] Gollac M, Volkoff S. Citius, altius, fortius, l'intensification du travail. Actes Rech Sci Soc 1996;114:54-67.

[25] Dejours C. L'évaluation du travail à l'épreuve du réel. Critique des fondements de l'évaluation. Paris: INRA; 2003.

[26] Molinier P. Les enjeux psychiques du travail. Paris: Payot et Rivages; 2006.

[27] Dejours C. Souffrance en France. La banalisation de l'injustice sociale. Paris : Seuil ; 1998. 